\title{
Nuclear-chemical processes in the solar atmosphere
}

\author{
Timashev Serge $e^{1,2}$ \\ ${ }^{1}$ Karpov Institute of Physical Chemistry, Moscow, Russia \\ ${ }^{2}$ Institute of Laser and Information Technologies, Russian Academy of Sciences, Moscow, Russia
}

Email address:

serget@mail.ru

\section{To cite this article:}

Timashev Serge. Nuclear-Chemical Processes in the Solar Atmosphere. International Journal of Astrophysics and Space Science. Vol. 2, No. 6, 2014, pp. 88-92. doi: 10.11648/j.ijass.20140206.12

\begin{abstract}
The ideas put forward earlier about the initiation of nuclear processes in a low-temperature plasma as a result of interaction between electrons of high (on chemical scales) energies and nuclei are demonstrated to be helpful in clearing some debatable issues concerning the synthesis of light elements in the solar atmosphere. Specifically the case in point is one of the puzzles associated with the radioactive isotope beryllium-7 whose abundance in the solar atmosphere exceeds the concentration of the stable isotope lithium-7 that is formed from beryllium-7, too, upon $K$-electron capture with a half-life of 53 days.
\end{abstract}

Keywords: Nuclear-Chemical Processes, Synthesis of Elements in the Solar Atmosphere

\section{Introduction}

According to the notions of the Standard Model of the Big Bang nucleosynthesis $[1,2]$, the first stage in the synthesis of elements is associated with the first three minutes after the Big Bang, when the first group of light elements $-\mathrm{H}, \mathrm{D}, 3 \mathrm{He}$, $4 \mathrm{He}$, and $7 \mathrm{Li}$ - was formed. (Versions involving the additional synthesis of $9 \mathrm{Be}$ and $11 \mathrm{Be}$ are also sometimes being discussed.) The second stage in the synthesis of elements, which had begun with the emergence of the first stars and has continued up to now, is characterized by nuclear reactions between elements in stars, as a result of which the elements up to those of the iron group $[2,3]$ in the periodic table have been formed. The further synthesis of more complex nuclei then becomes impossible, because the iron group nuclei have the highest binding energy per nucleon (around $8 \mathrm{MeV}$ ). And the third stage of the synthesis of elements is associated with supernova explosions. The Type I supernova explosions give birth to the elements from $\mathrm{Si}$ to $\mathrm{Fe}$ (the relative abundance of the elements from $\mathrm{O}$ to $\mathrm{Mg}$ changes but little), and it is only in explosions of the more massive Type II supernovae that those elements are formed whose mass exceeds that of the iron nucleus. The decisive role in these processes is assigned to $(n, \gamma)$ reactions and $\beta$-decays. The neutrons for such processes are formed in the reactions ${ }_{10}^{22} \mathrm{Ne}(\alpha, \mathrm{n}){ }_{12}^{25} \mathrm{Mg}$ and ${ }_{6}^{13} \mathrm{C}(\alpha, \mathrm{n}){ }_{8}^{16} \mathrm{O}$. If the parent nucleus, after its having captured a neutron and thus increased its mass number by unity, suffers a $\beta$-decay (the case of low neutron density or relatively high $\beta$-decay rates of the nuclei formed upon neutron capture), such a nuclear mass enhancement process is referred to as an s-process (slow mass growth). But in conditions of high neutron density or relatively low $\beta$-decay rates of the nuclei formed upon neutron capture, where the nucleus prior to its $\beta$-decay have enough time to capture a few neutrons, such a process is called an r-process (rapid mass growth). There is also a third process (the so-called p-process) wherein neutron-poor nuclei are formed, specifically as a result of $(p, n)$ or $(\gamma, n)$ reactions. Anyhow all the existing nucleosynthesis models focus mainly on the ideas that elements are synthesized through nuclear collisions, which requires such kinetic energies as can be realized in conditions of high-temperature plasmas obtaining in the internal regions of stars or in supernova explosions.

However, such ideas cannot help comprehensively to understand a number of anomalies that were revealed when analyzing the abundances of a number of light elements in our Galaxy and in the intergalactic space. To illustrate, according to Kramarovskii and Chechev [1], today the situation with the evaluation of the $\mathrm{D} / \mathrm{H}$ concentration ratio fixed in various regions of the Universe is not unambiguous. In particular, it has proved difficult to understand the spread of the $\mathrm{D} / \mathrm{H}$ values (which can be as great as an order of magnitude) measured in the local interstellar medium and also found from absorption lines of various quasars. It is 
usually believed that deuterium formed during the course of the original nucleosynthesis and existing in the primordial stellar medium gradually decays while it is participating in the reaction $\mathrm{D}(\mathrm{h}, \gamma) 3 \mathrm{He}$; and to gain an insight into the differences between the $\mathrm{D} / \mathrm{H}$ ratios measured, various mechanisms have been suggested for the formation of deuterium in galactic objects. However, not a single model suggested has won widespread recognition [1]. Serios problems are also associated with the high relative abundance of the isotope $3 \mathrm{He}$ fixed in glactic planetary nebulae. The $3 \mathrm{He} / \mathrm{He}$ ratio in these objects can reach $10-3$, which is two times as high as that in the galactic interstellar medium. To resolve such problems, one has to introduce some mechanisms for the formation of extra $3 \mathrm{He}$ after termination of the cosmological nucleosynthesis, which as yet seem quite controversial [1].

No less difficult to understand are the data on the dynamics of short-term variations in the relative abundances of other light elements and the variation dynamics of the isotope compositions of such elements in the atmosphere of stars accessible for analysis, our Sun in the first place. Specifically one of the puzzles of the Sun concerns the radioactive isotope beryllium-7 whose abundance in the solar atmosphere exceeds the concentration of the stable isotope lithium-7 that is formed from beryllium-7, too, upon $\mathrm{K}$-electron capture with a half-life of 53 days. At that the ratio $\eta={ }_{4}^{7} \mathrm{Be} /{ }_{3}^{7} \mathrm{Li}$ varies: it increases as the solar activity grows in intensity and decreases somewhat, remaining grater than unity, when the solar activity is at its minimum [4], which can be understood if beryllium-7 is being constantly formed in the solar atmosphere. Moreover, according to [5, $6]$, the isotope $7 \mathrm{Be}$ formed in the solar atmosphere contributes perceptibly to the radioactivity of the near-earth atmosphere, the concentration of this isotope in the air depending on the solar activity and featuring characteristic seasonal and latitude variations.

This isotope is customarily believed to be formed in the regions of powerful solar bursts as a result of nuclear processes involving protons accelerated in these regions and carbon, nitrogen, oxygen, and iron nuclei, and also as a consequence of fusion of the helium- 4 and helium-3 nuclei, because the concentration of helium- 3 in the regions of powerful solar bursts can prove comparable with that of helium-4 [7]. Of course, there remain questions as to the possibility of quantitative verification of such inferences when analyzing the dynamics of nuclear processes taking place in the solar atmosphere. One should also bear in mind the relatively low abundance of the above-mentioned elements (carbon, nitrogen, oxygen, and iron) in the solar atmosphere and also the low probability of formation of beryllium-7 in the reactions under discussion, which depends not only on the Coulomb barriers, but also on the threshold energies for these processes that exceed 10-25 MeV. The only above-threshold process here is the synthesis of beryllium-7 in the fusion of the helium- 3 and helium- 4 nuclei, but this reaction can be suppressed altogether because of the elevated Coulomb barrier.

\section{Initiation of Nuclear Reactions in the Inelastic Scattering of Electrons by Nuclei Via the Weak Interaction}

Fresh possibilities to understand the entire range of the above debatable issues concerning the abundance of light elements in stellar systems and interstellar medium can be offered by the ideas [8] that nuclear processes can be initiated in a low-temperature plasma upon interactions between nuclei and electrons with kinetic energies Ee up to some $5 \mathrm{eV}$, when inelastic scattering via the weak interaction channel involving the formation of the neutrino-antineutrino pair $v \tilde{v}$ can still remain unsuppressed. The threshold energy for such processes is around $0.3 \mathrm{eV}$. It was demonstrated in [8-10] that in the near-surface layers of Pd and $\mathrm{W}$ cathodes under glow discharge conditions $[9,11-13]$ and also in a low-temperature plasma formed in the near-surface regions of metals subject to laser ablation in ordinary and heavy water solutions [10,14-18] there can be initiated processes of nuclear synthesis of light elements and acceleration of radioactive nuclei decay. Attention was drawn to the fact that in the first stage of inelastic scattering via the weak interaction channel, after the neutrino $v$ was emitted and a virtual $\mathrm{W}$ - boson embedded in the nuclear matter, there must have been formed nuclei whose nuclear matter turned out to be in an unbalanced metastable state referred to as an "in-shake-up" state [8]. Indeed, the vector W- boson interacting with a u-quark of one of protons can bring about only the formation of a virtual d-quark and the subsequent chain of virtual transformations of quarks with the participation of vector $\mathrm{W}$ bosons, no neutrons being formed because of the mass deficit of such a nucleus (we assume that $\mathrm{K}$-electron capture is impossible for the original nucleus). The local anomaly formed in the nuclear matter as a result of strong pion-exchange interactions can be transferred to other nucleons. The initiated chain of virtual quark transformations with the participation of vector $\mathrm{W}$ - bosons must terminate with the irreversible decay of the $\mathrm{W}$ - boson into an electron and an antineutrino. We will therefore refer to such nuclei with their nuclear matter in a metastable shake-up state as $\beta$-nuclei. For such nuclei, the standard conception of nuclear matter as an aggregate of interacting nucleons becomes meaningless.

It is important to note here that the very fact of formation of nuclear matter in a metastable shake-up state must lead to the loss of stability of the nucleus as a consequence of the Casimir-force-dominated interrelation between the inner region of the nucleus, as a structurally organized subsystem, and the base medium - the physical vacuum $[19,20]$. It can be expected that in a shake-up state resulting from the rearrangements suffered by the internal structure of the nucleus the boundary conditions of the third kind, under which the polarization region of the QED vacuum in the vicinity of the nucleus is associated with its stable internal state, will obtain no longer, so that this relation must be disturbed. It is therefore but natural to suppose that in this case, when calculating the Casimir forces formed by the QED vacuum, there must be realized the boundary conditions 
that were formerly used in the modeling of the surface of the nucleus in the form of a metal sphere [21, 22]. According to the calculations $[21,22]$, the vacuum energy of such a sphere is positive, so that the Casimir forces will no longer provide for the stability of the nucleus. The latter means that the natural relaxation of the shake-up state must terminate with its $\beta$-decay - the formation of the original nucleus involving the emission of a free electron e- and a free antineutrino $\tilde{v}$.

So, the interaction of an electron with a deuteron $\mathrm{d}+$ during laser ablation of metals in heavy water or in a deuterium-containing glow-discharge plasma must lead to the formation of a neutral $\beta$-radioactive nucleus in a shake-up state, namely, the $\beta$-dineutron 2 nisu:

$$
e_{\text {he }}^{-}+d^{+} \rightarrow{ }^{2} n_{\text {isu }}+v
$$

In the above expression, the subscript on the symbol of the original electron ("high energy" on chemical scales) points to the initiative character of the electron-deuteron interaction in the formation of the $\beta$-dineutron ${ }^{2} n_{\text {isu }}$ and the neutrino $v$. The nucleus ${ }^{2} n_{\text {isu }}$ has a baryon number equal to two, zero leptonic charge, and a mass practically equal to the mass of the deuterium atom. The half-life $T_{1 / 2}$ of the $\beta$-dineutron that decays into deuteron, electron, and antineutrino,

$$
{ }^{2} n_{\text {isu }} \rightarrow d^{+}+e^{-}+\tilde{v}
$$

turns out to be long enough, no less than a few tens of seconds. This conclusion follows from the analysis of the experimental data on the synthesis of the tritium nuclei $t^{+}$in the laser ablation of metals in heavy water [18]. The latter process was supposed to take place upon interaction of the deuterium nucleus $d^{+}$with the nucleus ${ }^{2} n_{\text {isu }}$ :

$$
d^{+}+{ }^{2} n_{\text {isu }} \rightarrow t^{+}+n+Q(3.25 \mathrm{MeV})
$$

where $n$ is the neutron. Along with this process (3) there can also take place the process

$$
d^{+}+{ }^{2} n_{\text {isu }} \rightarrow{ }_{2}^{3} \mathrm{He}+n+e^{-}+\tilde{v}+Q(3.27 \mathrm{MeV})
$$

governed by weak nuclear interaction.

Postulated in [8] was also the formation of the hypothetical $\beta$-neutron $n_{\text {isu }}$ (upon interaction between electrons and protons $p^{+}$) and $\beta$-trineutron ${ }^{3} n_{\text {isu }}$ :

$$
\begin{aligned}
& p^{+}+e_{\mathrm{he}}^{-} \rightarrow n_{\mathrm{isu}}+v \\
& t^{+}+e_{\mathrm{he}}^{-} \rightarrow{ }^{3} n_{\mathrm{isu}}+v
\end{aligned}
$$

The rest masses of the nuclei introduced were taken to be equal to the rest masses of the hydrogen and tritium, respectively. It is precisely via the formation of the nucleus ${ }^{3} n_{\text {isu }}$ by the $e^{-}$-catalysis mechanism [8] that the process of initiated decay of tritium nuclei in conditions of laser ablation of metals in aqueous media, discovered in [18] along with the synthesis of tritium nuclei, can take its course:

$$
t^{+}+e_{\mathrm{he}}^{-} \rightarrow{ }^{3} n_{\mathrm{isu}}+v \rightarrow{ }_{2}^{3} \mathrm{He}+2 e^{-}+v+2 \tilde{v}+Q(0.019 \mathrm{MeV})
$$

It should be noted here that the half-life $T_{1 / 2}$ of the $\beta$-trineutron in the $e^{-}$-catalysis process proves, in accordance with [18], to be of the same order of magnitude as that of the nucleus ${ }^{2} n_{\text {isu }}$, which is many orders of magnitude shorter than the half-life of the tritium nucleus, $T_{1 / 2}=12.3$ years.

\section{Examples of Nuclear-Chemical Processes in the Solar Atmosphere}

Actually introduced within the scope of the ideas being expounded, which do not fit in with the existing notions of the synthesis of elements in the Universe [1-3], is a broad class of nuclear-chemical processes that can occur in conditions of low-temperature plasma. If protium, deuterium, and tritium nuclei are present in such a plasma and the nuclei ${ }^{b} n_{\text {isu }}$ are being formed there in accordance with processes (1), (4), and (5), where $b$ is the baryon number equal to 1,2 , and 3 for the $\beta$-neutron, $\beta$-dineutron, and $\beta$-trineutron, respectively, there can take place the processes

$$
\begin{aligned}
& { }_{Z}^{A} N+{ }^{b} n_{\text {isu }} \rightarrow{ }_{Z}^{A-A_{1}+k} N+Z_{1}{ }_{Z_{1}+1}^{A_{1}} N+(b-k) n+e^{-}+\tilde{v}+Q_{1} \\
& { }_{Z}^{A} N+{ }^{b} n_{\text {isu }} \rightarrow{ }_{Z}^{A-A_{1}+k} N+Z_{1}{ }_{Z_{1}}^{A_{1}} N+(b-k) n+Q_{1}
\end{aligned}
$$

where $k \leq b$.

It can be supposed that the $\beta$-neutron $n_{\text {isu }}$ and $\beta$-trineuron ${ }^{3} n_{\text {isu }}$, whose $\beta$-decay is governed by weak nteractions, should be sufficiently long-lived nuclei, like the $\beta$-dineutron ${ }^{2} n_{\text {isu }}$, so that the half-lives of the $\beta$-neutron and $\beta$-trineuron can be comparable with that of the $\beta$-dineutron. The latter conclusion suggests that the hypothetical nuclei $n_{\text {isu }},{ }^{2} n_{\text {isu }}$, and ${ }^{3} n_{\text {isu }}$ with their sufficiently long half-lives might participate in nuclear synthesis processes in stars. To illustrate, let us show some possible processes of formation of the deuterium, helium-3, and helium-4 nuclei, and also stable isotopes of light elements (from ${ }_{3}^{6} \mathrm{Li}$ to ${ }_{6}^{12} \mathrm{C}$ ) and the first elements more massive than iron:

$$
\begin{gathered}
{ }_{2}^{4} \mathrm{He}+{ }^{2} n_{\text {isu }} \rightarrow{ }_{3}^{6} \mathrm{Li}+e^{-}+\tilde{v}+Q(1.47 \mathrm{MeV}), \\
{ }_{2}^{4} \mathrm{He}+{ }^{3} n_{\text {isu }} \rightarrow{ }_{3}^{7} \mathrm{Li}+e^{-}+\tilde{v}+Q(2.47 \mathrm{MeV}), \\
{ }_{3}^{6} \mathrm{Li}+n_{\text {isu }} \rightarrow{ }_{3}^{7} \mathrm{Li}+\gamma(7.25 \mathrm{MeV}), \\
{ }_{3}^{7} \mathrm{Li}+{ }^{2} n_{\text {isu }} \rightarrow{ }_{4}^{9} \mathrm{Be}+e^{-}+\tilde{v}+Q(16.69 \mathrm{MeV}), \\
{ }_{4}^{9} \mathrm{Be}+n_{\text {isu }} \rightarrow{ }_{5}^{10} \mathrm{~B}+e^{-}+\tilde{v}+Q(7.36 \mathrm{MeV}), \\
{ }_{4}^{9} \mathrm{Be}+{ }^{2} n_{\text {isu }} \rightarrow{ }_{5}^{11} \mathrm{~B}+e^{-}+\tilde{v}+Q(17.81 \mathrm{MeV}), \\
{ }_{5}^{10} \mathrm{~B}+{ }^{2} n_{\text {isu }} \rightarrow{ }_{6}^{12} \mathrm{C}+e^{-}+\tilde{v}+Q(25.18 \mathrm{MeV}), \\
{ }_{26}^{56} \mathrm{Fe}+{ }^{2} n_{\text {isu }} \rightarrow{ }_{26}^{57} \mathrm{Fe}+n+Q(4.64 \mathrm{MeV}), \\
{ }_{26}^{56} \mathrm{Fe}+n_{\text {isu }} \rightarrow{ }_{26}^{57} \mathrm{Fe}+\gamma(7.64 \mathrm{MeV}), \\
{ }_{26}^{57} \mathrm{Fe}+{ }^{2} n_{\text {isu }} \rightarrow{ }_{27}^{59} \mathrm{Co}+e^{-}+\tilde{v}+Q(15.18 \mathrm{MeV}),
\end{gathered}
$$




$$
\begin{aligned}
& { }_{27}^{59} \mathrm{Co}+n_{\text {isu }} \rightarrow{ }_{28}^{60} \mathrm{Ni}+e^{-}+\tilde{v}+Q(10.31 \mathrm{MeV}), \\
& { }_{27}^{59} \mathrm{Co}+{ }^{2} n_{\text {isu }} \rightarrow{ }_{28}^{61} \mathrm{Ni}+e^{-}+\tilde{v}+Q(15.12 \mathrm{MeV}), \\
& { }_{28}^{62} \mathrm{Ni}+\mathrm{n}_{\text {isu }} \rightarrow{ }_{29}^{63} \mathrm{Cu}+\mathrm{e}^{-}+\tilde{v}+Q(6.12 \mathrm{MeV}) .
\end{aligned}
$$

The formation of the radioactive isotope beryllium- 7 in the solar atmosphere can be associated with the presence therein of the $\beta$-nuclei $n_{\text {isu }}$ and ${ }^{2} n_{\text {isu }}$, along with the nuclei ${ }_{3}^{6} \mathrm{Li}$ formed, as noted above, upon interaction between ${ }_{2}^{4} \mathrm{He}$ and ${ }^{2} n_{\text {isu }}$ :

$$
\begin{gathered}
{ }_{3}^{6} \mathrm{Li}+n_{\text {isu }} \rightarrow{ }_{4}^{7} \mathrm{Be}+e^{-}+\tilde{v}+Q(5.61 \mathrm{MeV}) \\
{ }_{3}^{6} \mathrm{Li}+{ }^{2} n_{\text {isu }} \rightarrow{ }_{4}^{7} \mathrm{Be}+n+e^{-}+\tilde{v}+Q(3.38 \mathrm{MeV})
\end{gathered}
$$

As for the above-noted problem of the excessive concentration of ${ }_{2}^{3} \mathrm{He}$ in the region of solar bursts [7], it is but natural to associate this phenomenon with the initiation of the processes of formation of helium-3 in accordance with reactions (3a) and (6). Inasmuch as the concentration of the nuclei ${ }^{3} n_{\text {isu }}$ can also increase in the region of solar bursts, the possibility cannot be ruled out that ${ }_{4}^{7} \mathrm{Be}$ can in that case be formed in the following process:

$$
{ }_{2}^{4} \mathrm{He}+{ }^{3} \mathrm{n}_{\mathrm{isu}} \rightarrow{ }_{4}^{7} \mathrm{Be}+2 \mathrm{e}^{-}+2 \tilde{v}+\mathrm{Q}(1.6 \mathrm{MeV})
$$

On the basis of the issue one can also understand the phenomenon of the relatively high concentration of the radioactive isotope beryllium-7 obtaining in the solar atmosphere and the relatively low concentration of lithium-6, which is an order of magnitude lower than the concentration of lithium-7 [4]. One should bear in mind here that the concentration of lithium-7 in the solar atmosphere depends on the solar activity of its own accord: it is at its maximum at the solar activity minima and a minimum at the solar cycle maxima [23]. This is usually being associated with the higher "burnout" rate of lithium-7 as a result of the process

$$
{ }_{3}^{7} \mathrm{Li}+\mathrm{p} \rightarrow 2{ }_{2}^{4} \mathrm{He}+\mathrm{Q}(13.35 \mathrm{MeV})
$$

that takes its course most intensively at the maximum of solar activity, when the number of powerful solar bursts increases. Insofar as doubts arise all the same in this case as well that process (11) can take place in view of the pertinent Coulomb barrier, it is quite natural to consider the above-noted alternative process of the more intense burnout of lithium-7 in the region of solar bursts upon its interaction with the $\beta$-nuclei ${ }^{2} n_{\text {isu }}$ involving the formation of beryllium-9. It is thus becomes possible qualitatively to understand both of the phenomena observed: the increase of the ratio $\eta={ }_{4}^{7} \mathrm{Be} /{ }_{3}^{7} \mathrm{Li}$ with increasing solar activity and its decrease to some extent (while remaining greater than unity) when the solar activity is at its minimum [4] and also the variation of the concentration of lithium-7 in the solar atmosphere from the minimal values (at the solar cycle maxima) to the maximal ones (at the minima of the solar activity) [23].

\section{Conclusion}

How far can the ideas expounded about the role of low-energy (on nuclear scales) extraneous effects on nuclear processes alter the traditional views on the synthesis of elements in the Universe? It has been demonstrated earlier that the probability of the $s$-processes of nucleosynthesis in stars is affected by the state of the electron shell of $\beta$-radioactive nuclei. In particular, in the case of complete ionization of the pertinent atoms, not only the probability of the $\beta$-decay of such nuclei can grow substantially, but their $\beta$-decay channels can alter $[24,25]$. At the same time, in the case of complete ionization of the pertinent atoms, the $\alpha$-decay probability remains unchanged [26]. However, within the scope of the notions expounded in this work, low-energy nuclear-chemical processes dominated by weak nuclear interactions can have a substantial effect on the dynamics of both $\alpha$ - and $\beta$-decay processes [9].

As demonstrated in [9], electrons in the deuterium-containing plasma of a gas-discharge setup with a tungsten cathode initiated $\alpha$-decay processes of five tungsten isotopes taken to be practically stable (the half-lives $T_{1 / 2}$ of all these isotopes comes to $10^{18}-10^{19}$ years), though their $\alpha$-decay is in principle possible. The initiation of the above processes takes place in the near-surface cathode layers of the gas-discharge setup upon interaction between tungsten isotopes and the metastable nuclei ${ }^{2} n_{\text {isu }}$. Since in addition to tungsten isotopes there are a great enough number of such "stable" isotopes capable of $\alpha$-decay, starting with neodymium and ending with bismuth, the examination of the above range of processes can prove important for the understanding of the entire scope of problems concerning the occurrence of elements in the Universe.

The results of direct experiments on the synthesis of heavy elements can apparently become decisive arguments allowing one to accept the notions expounded in this work, as well as in [8-10], about the role of electron-nuclear processes in the variation dynamics of the isotope composition of light elements in the solar atmosphere and also about the existence of the neutral nuclei ${ }^{b} n_{\text {isu. }}$. The case at hand are the processes of synthesis of cobalt and nickel from iron in the first place, and also of copper from nickel, in conditions of low-temperature plasma, including those in the case of laser ablation of metals or treatment of iron or nickel cathodes with a deuterium-containing glow-discharge plasma. As noted above, within the framework of the notions existing in the modern cosmology, such synthesis can only be possible in the Type II supernovae.

\section{References}

[1] Kramarovskii Ya.M., Chechev V.P. The puzzle of the lightest elements: observations, predictions, hypotheses // Phys. Usp. 1999. V. 42. N 6/ P. 563-572.

[2] Klapdor-Kleingrothaus H.V., Zuber K. Teilchenastrophysik. B.G. Teubner GmbH, Stuttgart, 1997.

[3] Bednyakov V.A. About creation of the chemical elements // Physics of Elementary Particles and Atomic Nuclei. 2002. part 4. P. 914-963 (in Russian). 
[4] Kuzhevskii B.M. Oject of research - the Sun // Journal Science in Russia. 2002. vol. 4. P. 4-11 (in Russian).

[5] Bezuglov M.V., Malyshevsky V.S., Malykhina T.V. et al. Photoproduction cosmogenic beryllium-7 in the earth's atmosphere // Electronic scientific journal "Studied in Russia" 2011. 589-591 (in Russian); http://zhurnal.ape.relarn.ru/articles/2011/046.pdf

[6] Buraeva E.A., Davydov M.G., Zorina L.V. et al. The content of cosmogenic Be-7 in surface air temperate latitudes //Atomic Energy. 2007. V.102. N 6. P. 370-374 (in Russian)

[7] Kocharov G.E. The puzzles of the Sun // The Soros Education Journal. 1998. N 3. P. 100-1004 (in Russian)

[8] Timashev S. F. Physical vacuum as a system manifesting itself on various scales - from nuclear physics to cosmology; http://arxiv.org/abs/1107.1799v7

[9] Savvatimova I.B., Timashev S.F. Nuclear-Chemical processes in the initiated tungsten and tantalum nuclei decay under actions of deuterium-containing low-temperature plasma to the cathodes of these materials // Condensed Matter Nucl. Sci., in press

[10] Timashev S. F., Simakin A. V., Shafeev G. A. Nuclear-Chemical processes under the conditions of laser ablation of metals in aqueous media (Problems of "cold fusion") // Russian Journal of Physical Chemistry A. 2014. V. 88. N. 11. P. $1980-1988$

[11] Savvatimova I.B., Gavritenkov D.V. In: Condensed Matter Nuclear Science. Proc. $12^{\text {th }}$ Intern. Conf. on Cold Fusion, Yokohamacity, Japan. 27 Nov-2 Dec 2005. World Scientific Publishing Co. Pte. Ltd.: Singapore, 231-252 p.

[12] Savvatimova I. Creation of more light elements in tungsten irradiated by low-energy deuterium ions. Proc.13th Int. Conf.ICCF13, Sochy, Russia, 2007. P. 505-517.

[13] Savvatimova I., Savvatimov G., Kornilova A. Decay in tungsten irradiated by low energy deuterium ions, Proc.13th Int. Conf.ICCF13, Sochy, Russia, 2007

[14] Shafeev G.A., Simakin A.V., Bozon-Verduraz F., Robert M. // Applied Surface Science. 2007. V. 254 P. 1022-1026.
[15] Simakin A.V., Shafeev G.A. // Applied Physics A. 2009. V. 101. N. 1. P. 199-203.

[16] Barmina E.V., Sukhov I.A., Lepekhin N.M. et al // Quantum Electronics. 2013. V. 43. N 6. P. 591-596 (in Russian).

[17] Simakin A.V., Shafeev G.A. // Physics of Wave Phenomena. 2008. V. 16. N. 4. P. 268-274.

[18] Barmina E.V., Kuzmin P.G., Timashev S.F., Shafeev G.A. / http://arxiv.org/abs/1306.0830

[19] Timashev S. Dynamical essence of the basic relations of the special theory of relativity and the origin of fundamental interactions: Phenomenology // International Journal of Astrophysics and Space Science, 2014. V. 2(2). P. 22-32.

[20] Timashev Serge. Planckian energy-mass source and the dynamics of the Universe: Phenomenology // International Journal of Astrophysics and Space Science, 2014. V. 2(3). P. $33-45$.

[21] J. Milton K.A., DeRaad L.L., Jr, Schwinger J. Casimir self-stress on a perfectly conducting spherical shell // Annals of Physics. 1978. V. 115. P. 388-403.

[22] Balian R., Duplantier B. Electromagnetic waves near perfect conductors. II. Casimir effect // Annals of Physics. 1978. V. 112. P. $165-208$.

[23] Baranovskii E.A., Musorina S.A., Taraschuk V.P. Барановский Э.А., Мусорина С.А., Таращук В.П. Determination of lithium in sunspots, the observations in 2007 // Proceedings of the Crimean Astrophysical Observatory. 2013. V.109. N. 1. P. 165-172 (in Russian).

[24] Jung M., Bosh F., Beckert K. et al. First observation of bound-state $\beta^{-}$decay // Phys. Rev. Lett. 1992. V. 69. N 15. P. 2164-2167.

[25] Bosh F.,Faestermann T., Friese J. et al. Observation of bound-state $\beta^{-}$decay of fully ionized ${ }^{187} \mathrm{Re}:{ }^{187} \mathrm{Re}-{ }^{187} \mathrm{Os}$ Cosmochronometry // Phys. Rev. Lett. 1996. V. 77. N 26. P. 5190-5193.

[26] Igashov S.Yu., Tchuvil'sky Yu.M. Electron shell and $\alpha$-decay //arxiv:1212.0419v1 [nucl-th]. 